\title{
The wear characteristics of wire rope used in the self-propelled orchard transport
}

\author{
Liu WeiWei ${ }^{1, a}$, Li ShanJun*, 2, b and Bao XiuLan ${ }^{2, c}$ \\ ${ }^{1}$ School of Mechanical and Electrical Engineering, Zhuhai city polytechnic, Zhuhai 519000, China; \\ ${ }^{2}$ College of Engineering, Huazhong Agricultural University, Wuhan 430070, China o.

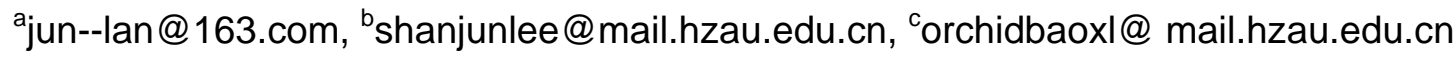

Keywords: wire rope, wear rule, local flaw, loss of metallic cross-sectional area.

\begin{abstract}
In order to ensure the stability of double track orchard self-propelled transport system and prevent tensile failure induced by the broken wire rope, we try to measure the state characteristics of the wire rope using the appropriate testing techniques and analysis methods, and monitor the wear condition of the wire rope according to the certain evaluation criteria. Considering the economic and operational convenience, the MTC computer wire rope flaw detector is used to detect the state and performance of the wire rope. After setting up and calibrating the parameters of the flaw detector, analyze the repeatability of the results of wire rope wear test, and then choose the LF and LMA data and curves of specific wire ropes to make comparisons. Results show that the testing method proposed in this paper is reproducible and reliable; the lifetime of a steel wire rope is about 3 - 4 years; the regular and gradual change of the broken wire are observed, and the broken wire position is concentrated; the wear also gradually increase and the largest section loss rate is up to $16.46 \%$.
\end{abstract}

\section{Introduction}

In order to solve the transportation problem in orchard, at present it has been developed from single, dual, walking tractor track and trackless and other types of Mountainous Orchard transport. The core technology of the transport lies in the drive section, of which the release-type power transmission device through the newly-invented driving wheel geared to the steel wire rope is to achieve drive based on the friction between the driving wheel and the steel wire rope. The purpose of the steel wire rope depends on its winding pattern on the driving wheel set: in-out of two ends of the steel wire rope is used for the automatically-running transport (As shown in Figure 1); while that of the same end of the steel wire rope for the trail-type transport (As shown in Figure 2).

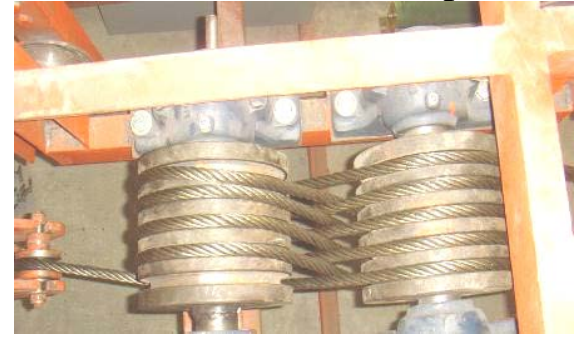

Fig.1 In-out of two ends of the steel wire rope

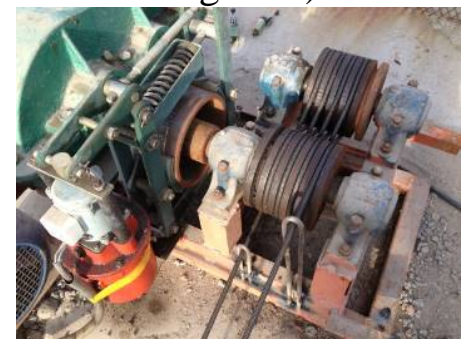

Fig.2 In-out of the same end of the steel wire rope

The adhesive wear and abrasive wear arising from the positive pressure between the external surface of the steel wire rope and the driving wheel as well as the curve fatigue wear and fretting wear from the interior of the steel wire rope may cause slipping for insufficient friction between the surface of the steel wire rope and the driving wheel or make the steel wire rope break and lose efficacy for fracture of the internal wire rope. Slipping will cause the transport cart not to function formally or in low efficiency but tensile failure for fracture of internal steel wire rope can lead to the disastrous accident. The wear condition of steel wire rope affects the safety and stability of the whole system, so it is necessary to conduct the detection and study of the wear characteristics of the steel wire rope.

The traditional detection method of steel wire rope is manual visual inspection, which is merely to inspect the external defects of the steel wire rope (for instance, broken wire) rather than the internal 
ones, and it can detect neither the invisible steel wire rope nor the section of the steel wire rope, and what's worse, the method is greatly affect by human factors and the reliability of the detection results is poor. The paper states that the steel wire rope undamaged detection method is used that in the situation of not damaging the function of the steel wire rope, the magnetization theory is employed to measure the wear features of the steel wire rope. Economic efficiency and operational convenience taken into account comprehensively, the MTC computer defect detector of steel wire rope is selected to stress the detection of LF(Local flaw) and LMA(Loss of metallic cross-sectional area) of steel wire rope in accordance with the national standard GB/T21837-2008 Practice for Electromagnetic Examination of Ferromagnetic Steel Wire Rope.

\section{Analysis of the Repeatability of Wire Rope Wear Test}

Configure the diameter, metallic cross-sectional area, lay length, sample interval, waveform magnification rate, cross-sectional sensitivity and other main parameters of the wire rope, and then verify the reliability of the flaw detector and the accuracy of parameter settings via repetitive testing. In order to avoid possible human errors during the wire rope testing, every wire rope test was carried out for more than five times. Results of three repetitive tests were shown in Table 1, Table 2 and Table 3 respectively.

Table 1 The first results for repetitive tests of wire breaking of the wire rope

\begin{tabular}{cccc}
\hline Sequence number & $\begin{array}{c}\text { Positions of broken } \\
\text { wires (Meter) }\end{array}$ & $\begin{array}{c}\text { Number of broken } \\
\text { wires }\end{array}$ & $\begin{array}{c}\text { Cumulative number } \\
\text { of broken wires } \\
\text { within the lay length }\end{array}$ \\
\hline 1 & 4.120 & 1 & 2 \\
2 & 4.155 & 1 & 2 \\
3 & 4.308 & 1 & 1 \\
4 & 4.401 & 1 & 2 \\
5 & 4.445 & 1 & 2 \\
6 & 4.477 & 1 & 1 \\
\hline
\end{tabular}

Table 2 The first results for repetitive tests of wire breaking of the wire rope

\begin{tabular}{cccc}
\hline Sequence number & $\begin{array}{c}\text { Positions of broken } \\
\text { wires (Meter) }\end{array}$ & $\begin{array}{c}\text { Number of broken } \\
\text { wires }\end{array}$ & $\begin{array}{c}\text { Cumulative number } \\
\text { of broken wires } \\
\text { within the lay length }\end{array}$ \\
\hline 1 & 4.044 & 1 & 2 \\
2 & 4.109 & 1 & 2 \\
3 & 4.143 & 1 & 1 \\
4 & 4.293 & 1 & 1 \\
5 & 4.393 & 1 & 1 \\
6 & 4.466 & 1 & 1 \\
\hline
\end{tabular}

Table 3 The third results for repetitive tests of wire breaking of the wire rope

\begin{tabular}{cccc}
\hline Sequence number & $\begin{array}{c}\text { Positions of broken } \\
\text { wires (Meter) }\end{array}$ & $\begin{array}{c}\text { Number of broken } \\
\text { wires }\end{array}$ & $\begin{array}{c}\text { Cumulative number } \\
\text { of broken wires } \\
\text { within the lay length }\end{array}$ \\
\hline 1 & 4.111 & 1 & 2 \\
2 & 4.143 & 1 & 1 \\
4 & 4.297 & 1 & 2 \\
5 & 4.393 & 1 & 2 \\
6 & 4.435 & 2 & 2 \\
7 & 4.466 & 1 & 1 \\
\hline
\end{tabular}

The waveforms of the three repetitive tests are shown as a, b and c in Figure 3. The red dots indicate where the wire breaking. The waveform amplitude at the red dot represents the number of broken wires. The greater the amplitude, the larger number of the broken wires.

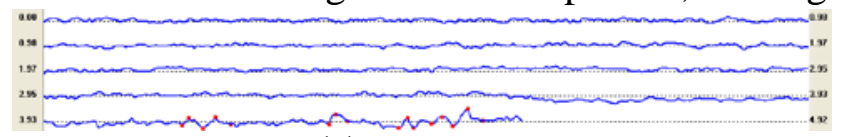

(a)

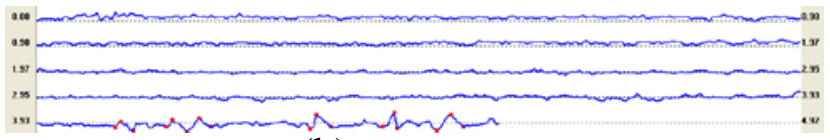

(b) 


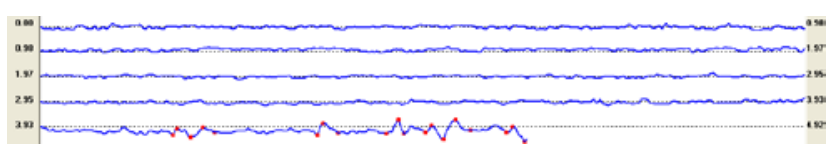

(c)

Fig. 3 Waveforms of the three repetitive tests for wire breaking of the wire rope

From the above testing data and waveform analysis, we can see that the test has good repeatability and the testing data is reliable. It is thus verified that the testing method and equipment can well detect the actual wear of wire ropes.

\section{Analysis of the Continuous Wear Test of Wire Rope}

The continuous wear test of wire rope is carried out in four groups. Each group operates the conveyor 200 times back and forth on the test platform and measures the actual wear of the wire rope after it goes through operation 200 times back and forth. To get the changes of the wire rope during the abrasion process, choose the LF(Local flaw) and LMA(Loss of metallic cross-sectional area) data and curves of one wire rope and make contrastive analysis respectively.

\subsection{Analysis of the Broken Wires}

\subsubsection{Analysis of the Number of Broken Wires}

After the wire rope goes through the four groups of wire rope wear tests, we can get the test results including the recorded number of broken wires, the total number of broken wires, the maximum number of broken wires, the maximum cumulative number of broken wires within the lay length and the positions of broken wires etc. The analysis result of experiment are shown in Table 4 and Figure 4.

Table 4 Analysis table of experiment result for wire breaking of the wire rope

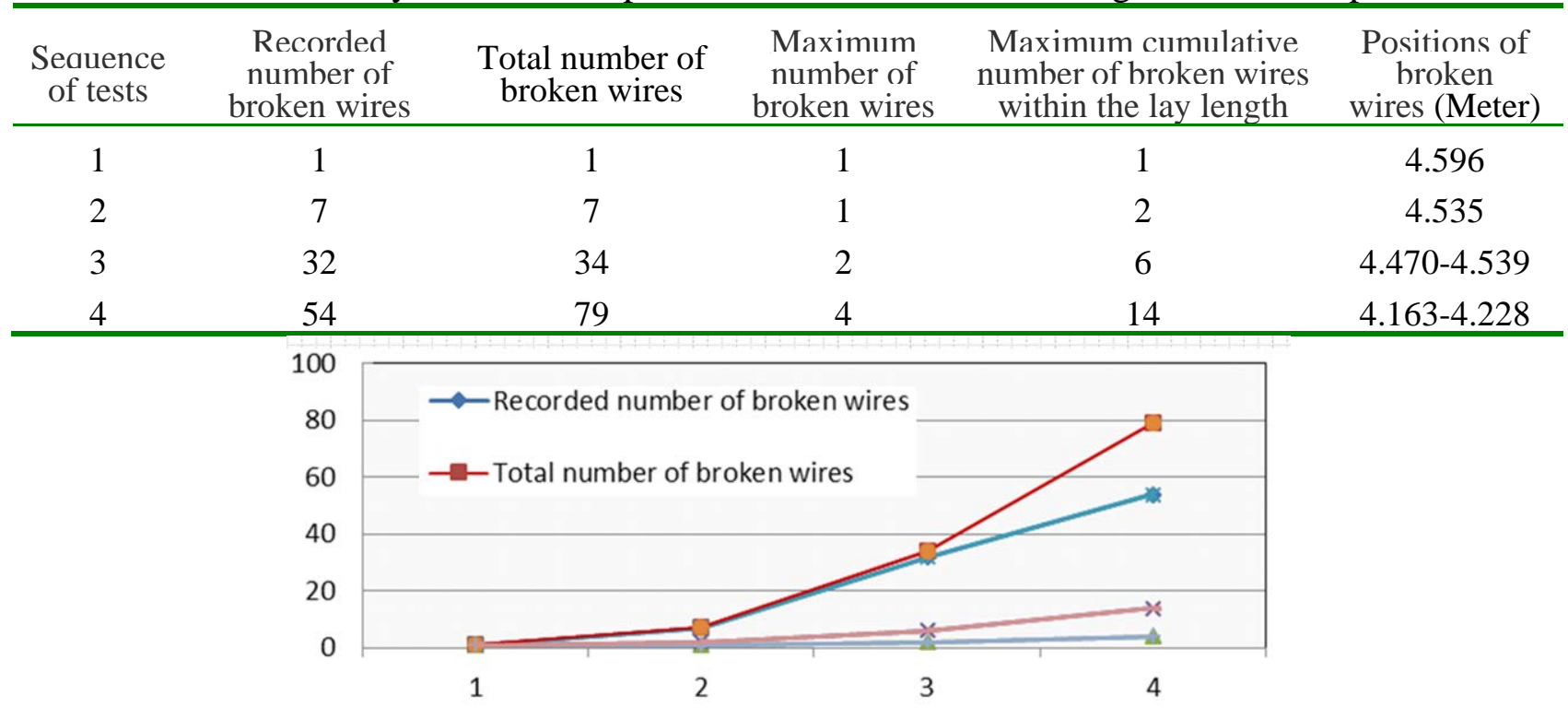

Fig.4 Analysis chart of experiment result for wire breaking of the wire rope

From the above table and figure, we can see that as the wear test proceeds, the total number of broken wires, the maximum number of broken wires, and the maximum cumulative number of broken wires within the lay length all shows an obvious increasingly upward trend. 


\subsubsection{Analysis of Waveforms of Broken Wires}

The waveforms of broken wires in the four tests are indicated by a, b, c and $d$ in Figure 5 . The red dots indicate where the wire breaking. The waveform amplitude at the red dot represents the number of broken wires. The greater the amplitude, the larger number of broken wires.

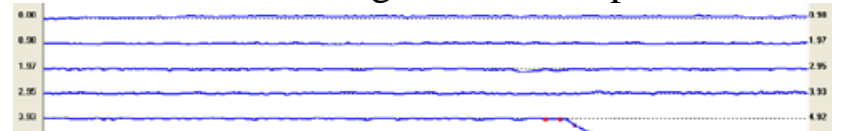

(a)

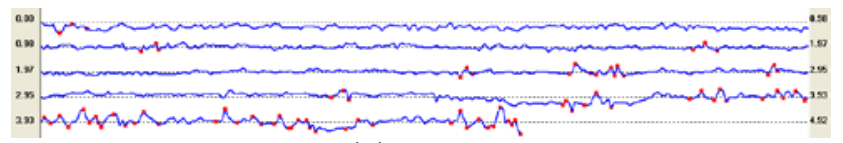

(c)

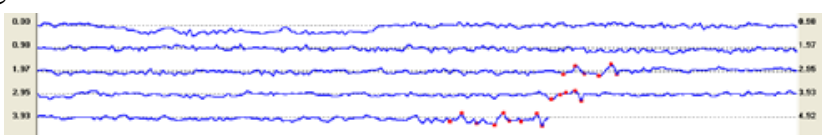

(b)

Fig. 5 Waveforms for broken wires of the wire rope in the four tests

From the waveforms, the change of broken wires shows good regularity and a gradual process, and the positions of broken wires are relatively concentrated.

\subsubsection{Analysis of the Physical Appearance of Broken Wires}

By observing and comparing with the real wires (as shown in Figure 6), we can find that the actual breaking phenomenon of wires is basically in line with the analytical graphs and tables of testing results and the analytical results of wire rope waveforms.

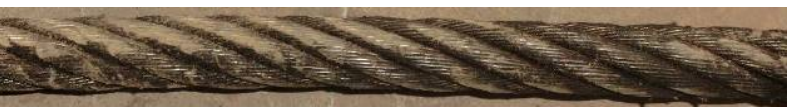

(a)

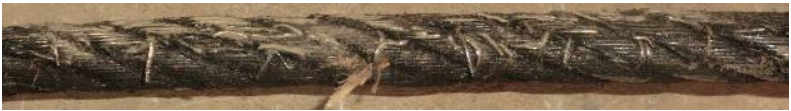

(c)

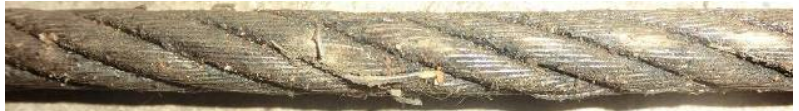

(b)

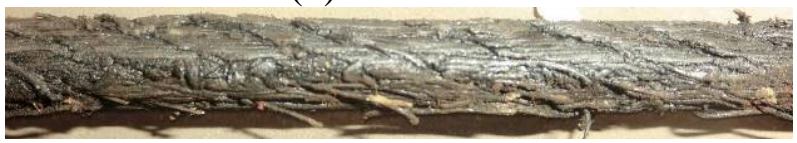

(d)

Fig. 6 Physical map for broken wires of the wire rope in the four tests

\subsection{Analysis of Wire Rope Wear}

Along with the occurrence of broken wires, internal and external wear of the wire rope is also occurring. The wear waveforms of the wire rope in the four tests are indicated by a, b, c and d in Figure 7.

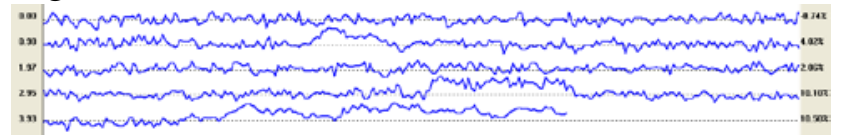

(a)

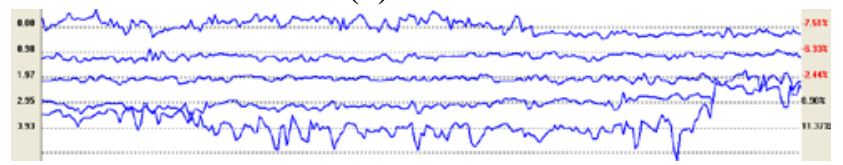

(c)

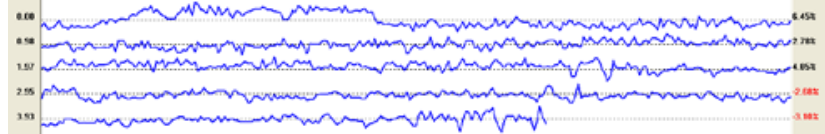

(b)

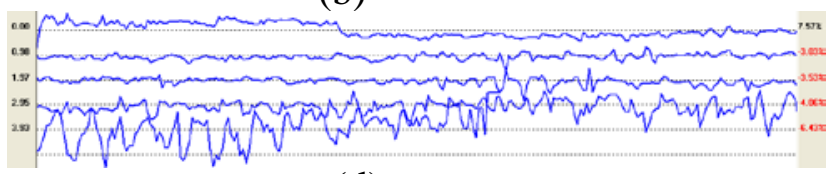

(d)

Fig.7 Wear waveforms of the wire rope in the four tests

The loss ratio of metallic cross-sectional area of wire rope is shown in Figure 8. The same to broken wires, the wear also shows the tendency of gradual exacerbation, with the maximum cross-sectional area loss rate of $16.46 \%$.

LMA(The loss ratio of metallic cross-sectionalarea)\%

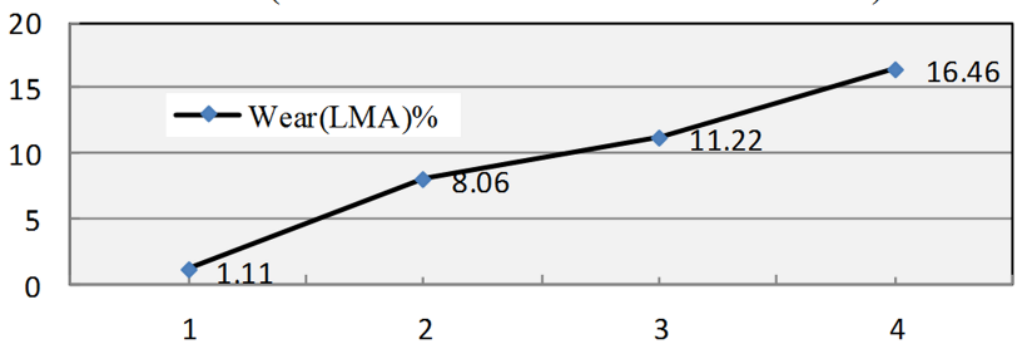

Fig.8 Loss ratio change for metallic cross-sectional area of wire rope 


\section{Prediction of Service Life of Wire Ropes}

Suppose that the transport aircraft is served in an orchard. The track is 200 meters long, with 100 meters on each side, and the orchard produces 2,500 kilograms fruits per mu; then the orchard needs to transport 75,000 kilograms fruits every year. Considering that the transport also needs to transport fertilizers, pesticides and other agricultural materials, which may add up to 25,000 kilograms annually, the conveyor thus has to support an annual transport capacity of 100,000 kilograms. The transport aircraft transports a volume of $500 \mathrm{~kg}$ a time, so it has to run 200 times back and forth annually. Therefore, when we carry out the wear test on the automatically controlled experimental platform for transport aircrafts, we take the operation 200 times back and forth on the platform as a period, which is equivalent to the transport capacity for one year. Before the test, we need to replace a new wire rope. The first wire rope is seriously damaged after three groups of testing, making the transport aircraft unable to operate. The second wire rope is seriously damaged after four groups of testing, making the transport aircraft unable to work. Therefore, it is estimated that a wire rope can be recycled for use $600 \sim 800$ times back and forth, which is equivalent to the service of three to four years of one wire rope according to the analysis aforementioned.

The test is based on the parameter standards (as shown in Table 5) regarding the number of broken wires of wire rope prescribed in the international standards ISO4309 Crane Wire Rope Inspection and Retirement Codes.

Table 5 Reference table of broken wires’ numbers of wire ropes used in steel pulley

\begin{tabular}{|c|c|c|c|c|c|c|c|c|c|}
\hline \multirow{4}{*}{$\begin{array}{l}\text { Number } \\
\text { of out-strands } \\
\text { bearing wire } \\
\text { (n) }\end{array}$} & \multirow{4}{*}{$\begin{array}{l}\text { Typical structure of } \\
\text { wire rope }\end{array}$} & \multicolumn{8}{|c|}{ Observable broken wires relative to fatigue strength of the wire rope on the crane } \\
\hline & & \multicolumn{4}{|c|}{$\begin{array}{c}\text { Mechaniral rlascification of M1, } \\
\text { M2, M3 and M4 }\end{array}$} & \multicolumn{4}{|c|}{$\begin{array}{l}\text { Mechanical classification of } \\
\text { M5, M6, M7 and M8 }\end{array}$} \\
\hline & & \multicolumn{2}{|c|}{ Interactive twist } & \multicolumn{2}{|c|}{ Lang lay } & \multicolumn{2}{|c|}{ Interactive twist } & \multicolumn{2}{|c|}{ Lang lay } \\
\hline & & $6 \mathrm{~d}$ & $30 \mathrm{~d}$ & $6 \mathrm{~d}$ & $30 \mathrm{~d}$ & $6 \mathrm{~d}$ & $30 \mathrm{~d}$ & $6 \mathrm{~d}$ & 30d \\
\hline $221 \leq \mathrm{n} \leq 240$ & $6 \times 37(18 / 12 / 6 / 1)$ & 10 & 19 & 5 & 10 & 19 & 38 & 10 & 19 \\
\hline
\end{tabular}

$\mathrm{d}$ is the nominal diameter of wire rope, $6 \mathrm{~d}$ is the lay length

This test selects a $6 \times 37$ interactive twist wire rope with $12 \mathrm{~mm}$ diameter. Therefore, according to the above standards, $6 \mathrm{~d}$ indicates that the maximum observable broken wires within the lay length are 10. The wire rope used in this test has 14 broken wires, the maximum number within the lay length after we complete the fourth test, which reaches the retirement standard. The result is in consistent with the actual situation.

\section{Conclusion}

(1) From the data and waveforms of the three repetitive tests of broken wires, we can see that it has good repeatability and produce reliable data to select MTC wire rope flaw detector to measure the wear regularity of wire rope.

(2) By analyzing the LF and LMA datas and curves of the four groups of wire rope wear tests, we can conclude that as the wear test proceeds, positions of broken wires become more concentrated. The total number of broken wires, the maximum number of broken wires, and the maximum cumulative number of broken wires within the lay length all shows an obvious increasingly upward trend. Along with the occurrence of broken wires, internal and external wear of the wire rope is also occurring. Its metal cross-sectional area loss rate also shows the tendency of gradual exacerbation, with the maximum cross-sectional area loss rate of $16.46 \%$.

(3) After the fourth test is completed, the wire rope used in this test has 14 broken wires, the maximum number within the lay length, which reaches the retirement standard and is in line with the actual situation. We take the operation 200 times back and forth on the platform as a period and estimate the service life of the wire rope, that is, a wire rope can be recycled for use 600 800 times back and forth, which is equivalent to the service of three to four years of one wire rope.

(4) In the process of wear test, only four groups of tests are carried out and only the wire rope is replaced due to the long test time. We do not replace the driving wheel, change the structure parameters, or test the wear of operating parameters, which all needs further and deeper researches. 


\section{Acknowledgements}

This study was supported by Natural Science Foundation of Hubei Province, China (Grant No.2014CFB322, No.201403036), Fundamental Research Funds for the Central Universities of China (Grant No. 2013QC007), Modern agricultural (citrus) industry technical system scientist position.

\section{References}

[1] M. Carlo, B. Silvia. Failure analysis of a cableway rope, Engineering Failure Analysis . 16(2009) 1666-1673

[2] D. Elata, R. Eshkenazy, M.P. Weiss. The mechanical behavior of a wire rope with an independent wire rope core, International Journal of Solids and Structures. 41(2004)1157-1172

[3] D.K. Zhang, S.R. Ge, Y.H. Qiang. Research on the fatigue and fracture behavior due to the fretting wear of steel wire in hoisting rope, Wear. 255(2003)1233-1237

[4] J.A. Hawk, R.D. Wilson, J.H. Tylczak, etc. Laboratory abrasive wear tests: investigation of test methods and alloy correlation, Wear.225 (1999)1031-1042

[5] J.J. Xing, S.J. Li, Y.L. Zhang. Main Parameters Calculation and Performance Test of 7YGS-45 Type Self-propelled Dual-track Orchard Transport, 2011 International Conference on Opto-Electronics Engineering and Information Science. 23 (2011)3025-3029

[6] M. Giglio, A. Manes. Life prediction of a wire rope subjected to axial and bending loads , Engineering Failure Analysi. 12(2005)549-568

[7] S.J. Li, J.J. Xing. Construction of 7YGS-45 Type Orchard Transport Automatic Control Test Platform, Advanced Materials Research.201 (2011)1936-1041

[8] J.F Zhang, J.Y. Li, Y.L. Zhang, S.J Li, M. Liang. Design of Remote Control Monorail Transporter for Mountainous Orchard, Transactions of the Chinese Society for Agricultural Machinery. 43(2012) 90-95

[9] S.J. Li, J.J. Xing, Y.L. Zhang, L. Meng, Q.Z. Fan. 7YGS- 45 Type Self-propelled Dual-track Mountain Orchard Transport, Transactions of the Chinese Society for Agricultural Machinery. 42(2011)85-88

[10]S.J. Li, J.J. Xing, etc. Construction of automatic test platform for 7YGS-45 Type Orchard Transport, The academic conference of Chinese Society of Agricultral Engineering in 2011. 22(2011)56-69

[11] W.J. Xu, H.Y. Cheng, G.L. Li, C.Y. Zhao. Wear mechanism analysis of inclined shaft hoisting steel , Journal of Hei Long Jiang institute of science and technology.18(2008) 407-410

[12] D.K. Zhang, S. Ge, Z.C. Zhu. Research on Evaluation of Fretting Parameters of Steel Wires, Journal of China university of Mining and Technology. 33(2004)33-36 